\title{
Glutamine blockade induces divergent metabolic programs to overcome tumor immune evasion
}

\author{
Robert D. Leone', Liang Zhao', Judson M. Englert', Im-Meng Sun', Min-Hee Oh', Im-Hong Sun', \\ Matthew L. Arwood', Ian A. Bettencourt', Chirag H. Patel', Jiayu Wen', Ada Tam', Richard L. Blosser', \\ Eva Prchalova ${ }^{2}$, Jesse Alt ${ }^{2}$, Rana Rais ${ }^{2}$, Barbara S. Slusher ${ }^{2}$, Jonathan D. Powell* \\ ${ }^{1}$ The Bloomberg-Kimmel Institute for Cancer Immunotherapy at Johns Hopkins, Baltimore, MD 21287, USA. ${ }^{2}$ Johns Hopkins Drug Discovery, Johns Hopkins School of \\ Medicine, Baltimore, MD 21205, USA. \\ *Corresponding author. Email: poweljo@jhmi.edu
}

The metabolic characteristics of tumors present significant hurdles to immune cell function and cancer immunotherapy. Using a novel glutamine antagonist, we metabolically dismantled the immunosuppressive microenvironment of tumors. We demonstrate that glutamine blockade in tumor-bearing mice suppresses oxidative and glycolytic metabolism of cancer cells, leading to decreased hypoxia, acidosis, and nutrient depletion. In contrast, effector $T$ cells responded to glutamine antagonism by markedly upregulating oxidative metabolism and adopting a long-lived, highly-activated phenotype. These divergent changes in cellular metabolism and programming form the basis for potent anti-tumor responses. Glutamine antagonism therefore exposes a previously undefined difference in metabolic plasticity between cancer cells and effector $T$ cells that can be exploited as a "metabolic checkpoint" for tumor immunotherapy.

Cancer cells have prodigious anabolic and energetic requirements and employ distinct metabolic pathways for growth and survival $(1,2)$. Termed the Warburg effect, cancer cells are highly glycolytic, metabolizing glucose to lactic acid to produce ATP and regenerate $\mathrm{NAD}+(1,3)$. In addition to glucose, glutamine plays an important role in providing both carbon and nitrogen necessary for anabolic metabolism (3, 4). As glucose is metabolized through glycolytic pathways to lactic acid, glutamine can fuel the TCA cycle, generating metabolic intermediates to serve as building blocks for lipids, proteins and nucleic acids that are crucial to anabolic growth and proliferation. Notably, these same metabolic programs have also been attributed to facilitating the tremendous growth associated with $\mathrm{T}$ cell activation and proliferation $(5$, 6). An important consequence of the specialized metabolism of cancer cells is the creation of a hypoxic, acidic, nutrientdepleted tumor microenvironment (TME) that is hostile to anti-tumor immune responses (7-14). In other words, the specialized metabolic programming of cancer cells not only promotes tumor growth but can also block the generation of an effective anti-tumor immune response.

We hypothesized that blocking glutamine metabolism would not only inhibit tumor growth, but also relieve a master immunometabolic checkpoint that enables restoration of antitumor immunity. Glutaminase facilitates the conversion of glutamine to glutamate, and the subsequent conversion of glutamate to $\alpha$-ketoglutarate represents a major source of glutamine into the TCA cycle $(1,3)$. However the therapeutic response of tumors in vivo to targeted blockade of glutaminase is generally limited (15-17). That is, inhibiting a single glutamine-requiring enzyme (glutaminase) proved to be minimally effective as tumors readily develop means to bypass this "targeted" therapy. Instead, we chose to comprehensively inhibit glutamine metabolism by using the glutamine antagonist 6-Diazo-5-oxo-L-norleucine (DON). In addition to glutaminase, DON inhibits a broad range of glutamine-requiring enzymes (18). Consequently, DON exposure potently inhibits tumor cell viability, proliferation, and cell cycle progression (fig. S1, A to E). Indeed, DON has previously been evaluated in a number of clinical trials for cancer, but these pursuits were eventually abandoned secondary to unacceptable toxicity, particularly to the gastrointestinal tract (19-24). Given the extraordinary potential of DON to not only inhibit tumor growth but also enhance immunotherapy, we have developed a series of novel prodrugs of DON that are designed to circulate intact and inert but are activated in the TME upon enzymatic cleavage of the promoieties, thus mitigating the previously reported toxicity of glutamine antagonists on susceptible tissues such as the gut (fig. S2, A and B) $(19,25)$. Of note, while we do observe an enrichment of the active, parent compound, DON, in tumor over plasma in our mouse model (fig. S2C), these prodrugs are much more rapidly activated in rodents compared with larger animals (2527). Nonetheless, we established a robust therapeutic window for in vivo mouse studies using the compound JHU083 to explore whether tumor glutamine metabolism can be targeted as an immunologic checkpoint (fig. S2, A to C) $(19,25,28-30$ ).

To examine the ability of JHU083 to inhibit tumor 
growth, we performed a series of experiments using syngeneic tumor models. Mice were injected subcutaneously with MC38 colon cancer, EL-4 lymphoma, CT26 colon cancer or B16 melanoma. For each tumor type tested, treatment with JHU083 led to a marked decrease in tumor growth and improved survival (Fig. 1A and fig. S2, D to F). In the case of MC38, JHU083 monotherapy for 14 days led to a significant proportion of durable responses. Given the ability of JHU083 to cause tumor regression, we used an in vivo model to examine the specific effects on tumor metabolism. Using ${ }^{13} \mathrm{C}$-glucose tracing with mass spectrometry, we observed differential enrichment of glucose metabolites in metabolic pathways. Blocking glutamine metabolism inhibited glucose metabolism through the TCA cycle, as well as through glycolysis and related pathways, thus disabling Warburg physiology (Fig. 1B and fig. S2G). We also noted a significant decline in glucose contribution to purine synthesis, the hexosamine pathway and the pentose phosphate pathway (Fig. 1B). This broad suppression of metabolic activity is accompanied by a significant increase in glutamine and glucose content within tumors (Fig. 1C), as well as a decrease in tumor hypoxia (Fig. 1D). Such findings suggest that blocking glutamine metabolism can critically disrupt the metabolism of the tumor as a whole, with marked effects on the nutrient milieu within the TME.

Given the pronounced changes observed in the TME, we asked whether targeting glutamine metabolism would enhance the efficacy of immunotherapy. We were initially concerned that blocking glutamine metabolism would severely impair lymphocyte function, thus metabolic treatment was administered prior to initiating adoptive cell transfer (ACT) immunotherapy. Mice harboring OVA-expressing B16 melanoma were pre-treated with JHU083 for 3 days before adoptive transfer of activated OVA-specific OTI T cells. Treated mice showed improvement in tumor control and survival (Fig. 1E), suggesting that glutamine blockade can condition the TME to enhance ACT. Next, we combined glutamine targeting with PD-1 checkpoint immunotherapy. Because it's possible that glutamine antagonism could suppress anti-tumor immune cells, we designed our initial treatment regimens to use sequential dosing of JHU083 for a defined period of several days, followed by anti-PD1 treatment. We found that the most efficacious regimens for inducing anti-tumor response required concurrent, not sequential, dosing of JHU083 and anti-PD-1. To this end, we found that tumorbearing mice treated with concurrent dosing of the glutamine antagonist and anti-PD-1 showed dramatically improved anti-tumor effects compared with anti-PD-1 therapy alone, with complete response rates approaching 100 percent in the MC38 model (versus no complete response in mice receiving anti-PD1 monotherapy) (Fig. 1F and fig. S2H). Importantly, these findings indicate that blockade of glutamine metabolism, despite the established role of glutamine for activated lymphocyte proliferation and function, does not metabolically disable anti-tumor immune cells, but to the contrary, appears to significantly enhance their function and effectiveness.

These findings, in addition to the observed ability of glutamine antagonism to promote durable tumor remissions as a single agent (Fig. 1A), led us to investigate the intriguing hypothesis that blocking glutamine metabolism alone could enhance endogenous anti-tumor responses, even in the absence of additional immunotherapy. To test this, we rechallenged mice that had been cured with JHU083 monotherapy with an equal burden of the same tumor injected on the opposite flank. Remarkably, almost all of the animals cured by single-agent JHU083 completely rejected the tumor upon rechallenge (Fig. 1G), suggesting the establishment of immunologic memory. To confirm the immunologic basis of this phenomenon, we compared JHU083 treatment of MC38bearing RAG2-/- mice and wild type mice. RAG2-deficient mice lack the VDJ recombinase machinery necessary for rearranging antigen receptor genes, and as such do not produce mature B or T lymphocytes and are incapable of mounting an adaptive immune response. While glutamine blockade with JHU083 had some initial effect on tumor growth in the RAG2-/- mice, tumor growth rates recovered after several days and the animals succumbed to the disease at a similar timeframe to untreated wild type mice (Fig. $1 \mathrm{H})$. Using T cell depleting antibodies (31), we were able to demonstrate that the efficacy of glutamine antagonist therapy was completely dependent upon the activity of the CD8+ T cell subset of the adaptive immune response (Fig. 1I). These observations demonstrate that glutamine blockade, without additional immunotherapy, markedly enhances endogenous anti-tumor immunity and, more generally, that tumor immune rejection and adaptive immune memory can be triggered solely through this metabolic intervention.

To further investigate the mechanism of this enhanced anti-tumor response to JHU083 monotherapy, we examined tumor infiltrating lymphocytes (TIL) from vehicle- and JHU083-treated mice bearing either MC38 or OVAexpressing MC38 tumors. OVA-expressing MC38 tumors allow identification and study of antigen specific CD8+ T cells by tetramer staining. Consistent with our depletion studies, we observed a marked increase in infiltrating CD8+ T cells with JHU083 treatment, and a greater percentage of these TIL were antigen specific (Fig. 2A). To explore the mechanism of the enhanced endogenous anti-tumor immunity, we performed RNA sequencing (RNA-seq) on FACS-sorted CD8+ TIL. RNA-seq revealed distinct differences between TIL from treated and untreated mice (fig. S3, A and B), with 4,313 genes differentially expressed in a statistically significant manner. Gene set enrichment analyses (GSEA) showed that CD8+ TIL from treated mice had transcriptional programs consistent 
with a highly proliferative (Fig. 2B), robustly activated (Fig. 2C), less exhausted and less anergic phenotype (fig. S3C). These transcriptional changes were also reflected at the protein level as FACS analyses of CD8+ TIL from MC38 tumors and of tetramer-positive CD8+ TIL from OVA-expressing MC38 tumors, show increased proliferation and activation markers (Fig. 2, B and C, and fig. S3D), decreased percentage of PD-1+LAG-3+ double positive TIL (fig. S3E) and enhanced IFNY (Fig. 2D), Granzyme B (Fig. 2D) and IL-2 (fig. S3F) production upon ex vivo stimulation. As was anticipated from our preliminary TME analysis, GSEA also suggested that CD8+ TIL in treated mice experience less hypoxia and oxidative stress (Fig. 2E and fig. S3G), which was corroborated by flow cytometry showing decreased pimonidazole (a marker of exposure to low oxygen tension) staining on CD45+ TIL (Fig. $2 \mathrm{E})$. Furthermore, we observed a relative upregulation of transcripts associated with long-lived, memory T cells (Fig. 2F) and a marked downregulation of apoptotic transcriptional programs in TIL from mice treated with glutamine blockade (Fig. 2G and fig. S3, H and I). Indeed, increased expression of memory markers (CD62L, CD127 CD122, and BCL6) (Fig. 2F and fig. S3, J and $\mathrm{K}$ ) and increased expression of anti-apoptotic protein, MCL-1 (Fig. 2G) were also observed by flow cytometry. We also confirmed by RT-PCR that apoptotic genes uncovered through leading edge GSEA analysis (fig. S3, $\mathrm{H}$ and I) were indeed downregulated in CD8+ TIL from JHU083-treated mice (fig. S3L). This downregulation has a tangible effect, as CD8+ TIL, or tetramer-OVA+ CD8+ TIL, were less susceptible to intratumoral cell death in JHU083treated mice (Fig. 2G, right). In accord with recent reports, these findings suggest that an important mechanism of CD8+ $\mathrm{T}$ cell dysfunction and tumor immune evasion may be apoptosis of tumor specific $\mathrm{T}$ cells within the TME (32). Blocking glutamine metabolism led to similar changes when combined with anti-PD-1 treatment (fig. S3, D, E, J, and K). Taken together, our results demonstrate that glutamine blockade conditions TIL toward a long-lived, memory-like phenotype that is highly proliferative, markedly activated and capable of enhanced effector function.

Our initial studies were motivated by the hypothesis that targeting tumor metabolism would alter the TME to enhance anti-tumor immune responses. Indeed, the decreased hypoxia of TIL in treated mice directly supports this mechanism (Figs. 1D and 2E). However, the striking changes observed in the activation and differentiation states of the TIL from treated and untreated mice led us to question whether glutamine antagonism was also directly affecting $\mathrm{T}$ cell activation and function. To assess the cell-intrinsic nature of these findings, we activated and expanded naïve CD8+ T cells with antiCD3, anti-CD28 and IL-2 in the presence or absence of DON (because JHU083 requires in vivo enzymatic cleavage of promoieties, DON is used for all in vitro studies). Much like our in vivo TIL analysis, in vitro activated T cells, in the presence of DON, showed robust upregulation of activation markers (CD44, CD69), memory markers (CD62L, CD127, CD122, CD27), anti-apoptotic proteins (BCL-2, MCL-1) and transcription factors (T-bet, EOMES, TCF-1, BCL-6) (Fig. 2, H to K). Moreover, restimulation of DON-treated CD8+ T cells reveals an increased capacity to produce cytokines and Granzyme B (Fig. 2L). These phenotypic changes are also observed when glutamine metabolism is inhibited in the context of acute infection with LCMV Armstrong (fig. S3, M to O). Transcriptional changes associated with a memory $\mathrm{T}$ cell phenotype have previously been reported in response to modulation of $\alpha$-ketoglutarate dependent demethylases, including critical epigenetic remodeling enzymes $(33,34)$. $\alpha$-Ketoglutarate is a proximal metabolite of glutamine metabolism. We observed significant attenuation of $\alpha$-ketoglutarate levels in DONtreated CD8 T cells 36 hours post activation (Fig. 2M), with increased methylation (corresponding to decreased activity of histone demethylase enzymes) on a broad range of histone methylation sites (Fig. 2N, left). Importantly, identical changes in histone methylation are evident on TIL from explanted tumors (Fig. 2N, right). These findings demonstrate that glutamine blockade, even in the absence of tumor, conditions activated $\mathrm{T}$ cells in a cell intrinsic manner toward a highly activated, long-lived, memory-like differentiation state.

Given the differential ability of glutamine metabolic inhibition to disable tumor cells while enhancing $\mathrm{T}$ cell function, we were interested in defining the cell-specific metabolic adaptations of cancer cells and CD8+ effector T cells in response to glutamine blockade. Metabolic flux analyses reveal that glutamine blockade by DON suppresses aerobic glycolysis (i.e., Warburg physiology) in cancer cells and activated CD8 T cells (Fig. 3, A and B, and fig. S4, A and B) as measured by extracellular acidification rate (ECAR). However, whereas DON profoundly suppresses oxidative phosphorylation (OXPHOS) in cancer cells, DON exposure during CD8 T cell activation triggers a dramatic upregulation of oxidative metabolism and spare respiratory capacity (Fig. 3, A and B, and fig. S4C). In DON-treated T cells, but not in MC38 cancer cells, these changes in metabolic programs are associated with upregulation of mitochondrial proteins TOMM20, CPT1 $\alpha$ and VDAC1 (Fig. 3, C and D). This is correlated with in vivo changes showing differential changes in expression of the same mitochondrial proteins in CD8+ TIL versus CD45negative tumor cells in the TME (Fig. 3E). Functional changes favoring OXPHOS (with increased SRC) over Warburg physiology in T cells are consistent with metabolic characteristics of a $\mathrm{T}$ cell memory phenotype and can be approximated by the OCR/ECAR ratio (35). In accord with memory-like phenotypic changes we observed in T cells exposed to JHU083 (Fig. 2, F, I, J, and K), we observed a markedly increased 
$\mathrm{OCR} / \mathrm{ECAR}$ ratio in both in vitro $\mathrm{T}$ cell experiments as well as in CD8+ TIL sorted from treated and untreated MC38bearing mice (Fig. 3F). To further explore the different metabolic responses in T cells and cancer cells, we were interested in determining the metabolic fuel for the increased OXPHOS in $\mathrm{T}$ cells undergoing glutamine blockade. Interestingly, we found that DON-treated $\mathrm{T}$ cells maintain dramatically elevated levels of OXPHOS despite pharmacologic inhibition of mitochondrial transport of long chain fatty acids, pyruvate and glutamine with etomoxir, UK0599 and BPTES, respectively (Fig. 3G). While metabolism of branched chain amino acids does not appear to be a significant source of fuel for DON-treated T cells (fig. S4D), ${ }^{13} \mathrm{C}$-tracing experiments show a dramatic ability of $\mathrm{T}$ cells to utilize acetate as a carbon source for the TCA cycle in the setting of glutamine blockade (Fig. $3 \mathrm{H}$ ). The unique ability of DON-treated T cells to metabolize acetate as a TCA fuel when compared to tumor cells is supported by the upregulation of Acyl-CoA Synthetase Short Chain Family Member 1 (ACSS1) and Acyl-CoA Synthetase Short Chain Family Member 2 (ACSS2) in vitro and in vivo (Fig. 3I and fig. S4E). These findings are in accord with recent reports of the importance of acetate in $\mathrm{CD} 8+\mathrm{T}$ cell function (36-38). In contrast, DON treated MC38 cells downregulate acetate metabolizing enzymes and show minimal incorporation of ${ }^{13} \mathrm{C}$-labeled acetate in TCA intermediates (Fig. 3, $\mathrm{H}$ and I). As such, acetyl CoA generation is reciprocally affected in MC38 vs CD8 T cells during glutamine blockade (Fig. 3J). Notably, downregulation of ACSS1 and ACSS2 in response to glutamine blockade was observed in multiple cancer cell lines, suggesting a general effect of glutamine blockade on cancer cell metabolism (fig. S4F).

Oxidation of acetyl-CoA through the TCA cycle is a central process in cellular energy metabolism. However, highly proliferative cells, such as activated effector T cells, also use TCA cycle intermediates as building blocks for a range of biomolecules, including nonessential amino acids, lipids and nucleic acids. Through a process called anaplerosis, these intermediates must be replenished to maintain TCA cycle function. Highly proliferative cells often fuel anaplerosis through glutamine metabolism, or glutaminolysis, which replenishes $\alpha$ ketoglutarate in the TCA cycle through glutaminase (GLS) activity. Because glutamine contribution to TCA intermediates (as well as other glutamine-requiring processes) is suppressed in DON-treated T cells and MC38 cells (Fig. 4A and fig. S5), we hypothesized that T cells employed an alternative anaplerotic source to maintain TCA activity. In this regard, pyruvate carboxylase (PC), which generates oxaloacetate from pyruvate, provides an important alternative anaplerotic pathway by allowing glucose to replenish TCA intermediates (39). As such, it has been reported that PC activity is required for cancer cell survival during targeted GLS inhibition (40). By performing stable-isotope tracing studies with glucose labeled with ${ }^{13} \mathrm{C}$ at all six carbons, we found that DON-treated MC38 cells show a profound suppression of glucose-derived carbons contributing to the TCA cycle, an effect which is not apparent in DON-treated T cells, nor in MC38 treated with the GLS inhibitor, CB839 (Fig. 4B and fig. S6A). In sharp contrast, DON-treated T cells show significant flexibility in glucose metabolism as they are capable of upregulating TCA entry of glucose-derived carbons both as acetyl-CoA, as well as oxaloacetate through PC-mediated anaplerosis. As such, the pattern of glucose carbons entering the TCA cycle in DON-treated T cells (and GLS-inhibited MC38 cells), including increased $\mathrm{M}+3$ isotopologues in citrate, malate, aspartate, and $\mathrm{M}+5$ isotopologue in citrate, is indicative of enhanced $\mathrm{PC}$ activity (Fig. 4, C and D, and fig. S6B) (41). Unlike DONtreated $\mathrm{T}$ cells, this signature is broadly attenuated in DONtreated MC38 cells (Fig. 4D). Notably, PC activity is critically dependent upon activation by acetyl-CoA $(42,43)$. In this regard, the differential activity of PC (which is expressed equally across each of these cell types and treatments) observed in these cells (fig. S6C), correlates with elevated acetylCoA levels in DON-treated T cells and decreased acetyl-CoA in DON-treated MC38 cells (Fig. 3J).

The inability of DON-treated MC38 cells to supply glucose-derived metabolites for the TCA cycle (Fig. 4C) or to maintain Warburg physiology (Fig. 3A) is likely due to the suppressive effects of DON on proximal glycolytic metabolism in these cells. Unlike DON-treated T cells and MC38 cells treated with CB839, DON-treated MC38 cancer cells exhibit decreased glucose uptake and diminished levels of early glycolytic intermediates (Fig. 4, E and F, and fig. S6D). We were interested in the mechanism of glycolysis suppression in MC38 cells treated with glutamine antagonism. Unlike T cells, wherein metabolic adaptations to DON treatment (including upregulated acetate metabolism and PC activity) allow them to maintain the cellular AMP/ATP ratio, MC38 cells are unable to compensate energetically, leading to dramatically increased AMP/ATP ratios and robust activation of AMP kinase (Fig. 4, G and H). Furthermore, MC38 cells exposed to glutamine blockade markedly downregulate c-MYC (Fig. 4H), a phenomenon previously reported in cancer cells in response to glutamine deprivation (44). We observed this effect in a range of murine cancer cell lines (fig. S4F). As c-MYC and AMPK are critical regulators of glycolytic flux (45-47), the downregulation of c-MYC and concurrent activation of AMPK play crucial roles in the suppression of glycolytic pathways in DON-treated MC38 cells. Like DON-treated T cells, however, CB839-treated MC38 cells maintain c-MYC expression, AMP/ATP ratios, and show no increased AMPK signaling (fig. S6, C, E, and F).

Given the effects of glutamine antagonism on suppressing proximal glycolysis and Warburg physiology in MC38 cells, we hypothesized that parallel metabolic pathways may also 
be affected. We were particularly interested in the activity of the pentose phosphate pathway (PPP). The PPP is a branching pathway from glycolysis and is the major cellular source for NADPH production, which is vital for maintenance of redox homeostasis. In addition to their known role in regulating glycolytic flux c-MYC and AMPK also regulate the activity of the PPP $(46,48,49)$. Indeed, unlike DON-treated T cells and CB839-treated MC38 cells, DON-treated MC38 cells are unable to maintain NADPH/NADP+ homeostasis (Fig. 4I and fig. S6G), indicative of a defect in this pathway. This is further supported by measurement of the activity of the PPP rate limiting enzyme, G6PD, which is correspondingly suppressed in DON treated MC38 cancer cells but elevated in DON treated CD8+ T cells. (Fig. 4J).

While tracing experiments confirmed blockade of glutamine pathways during DON or JHU083 treatment (fig. S5), we were interested in assessing the specific contribution of key glutamine-utilizing enzymes to the observed metabolic phenotype (fig. S7A). We used shRNA lentiviral constructs to generate stable knockdowns of glutamine-utilizing enzymes in the MC38 cell line (fig. S7, B and C). We found that knocking down several of these genes individually (e.g., Gfpt1, Ctps1, Asns) contributed to c-MYC downregulation, reduced glucose uptake and suppressed proliferation (fig. S7, D to F). However, when each of these lines was treated with DON, further downregulation of c-MYC was observed (fig. S7D). That is, while several knockdown cell lines demonstrate suppressed glucose uptake, downregulated c-MYC and attenuated proliferation, no single gene completely accounted for the phenotype that was observed through inhibition of all glutamine-utilizing pathways simultaneously (with DON or JHU083) (fig. S7, D and G).

In contrast to cancer cells, activated CD8 $\mathrm{T}$ cells adapt to glutamine blockade through the upregulation of acetate metabolism, generating high levels of acetyl CoA to fuel the TCA cycle directly (as a 2-carbon source) and indirectly (as an activator of glucose anaplerosis through increased PC activity) (Fig. 4K). In addition to maintaining energy homeostasis (i.e., AMP/ATP ratio) (Fig. 4G), the ability of T cells to utilize acetate to fuel OXPHOS and produce ATP enables them to divert glucose into the PPP and maintain $\operatorname{NADP}(\mathrm{H})$ homeostasis (Fig. 4J). Overall, our studies demonstrate that for cancer cells, the interdependence of glycolysis, OXPHOS and glutamine metabolism lacks plasticity such that targeting glutamine metabolism leads to wide-ranging metabolic inhibition, disruption of $\mathrm{NADP}(\mathrm{H})$ homeostasis, and stymied growth. Conversely, targeting glutamine metabolism in $\mathrm{T}$ cells leads to adaptive metabolic reprogramming with enhanced survival, proliferation and effector function.

From the time of the pioneering work of Otto Warburg, the study of immune cell metabolism has been linked to our understanding of cancer cell metabolism as a paradigm for the behavior of highly proliferative cells $(5,50-54)$. While both activated T cells and cancer cells employ Warburg physiology and glutamine metabolism to fuel remarkably high proliferative rates $(2,54,55)$, the precise role of Warburg physiology, an inherently less efficient mode of ATP generation than oxidative respiration, has not been established. Recent theories have suggested that highly proliferative cells engage Warburg physiology to maintain the activity of critical pathways such as the PPP that emanate from the glycolytic pathway (56). We show here that, while increased glucose uptake may fuel increased PPP activity (as observed in DON-treated T cells), the function of this pathway does not require that glucose is metabolized to lactic acid, i.e., Warburg physiology. That is, the PPP runs in parallel with glycolysis, but is not necessarily dependent upon it. As such, in addition to establishing glutamine metabolism as a metabolic checkpoint for cancer immunotherapy, our studies suggest fundamental insights into the nature and role of Warburg physiology. Moreover, through the application of glutamine blockade, we demonstrate the possibility of differentially modulating the metabolism of cancer cells and anti-tumor immune cells by exploiting the inherent metabolic plasticity of each cell type.

\section{REFERENCES AND NOTES}

1. N. N. Pavlova, C. B. Thompson, The emerging hallmarks of cancer metabolism. Cell Metab. 23, 27-47 (2016). doi:10.1016/i.cmet.2015.12.006 Medline

2. A. J. Wolpaw, C. V. Dang, Exploiting metabolic vulnerabilities of cancer with precision and accuracy. Trends Cell Biol. 28, 201-212 (2018). doi:10.1016/j.tcb.2017.11.006 Medline

3. B. J. Altman, Z. E. Stine, C. V. Dang, From Krebs to clinic: Glutamine metabolism to cancer therapy. Nat. Rev. Cancer 16, 619-634 (2016). doi:10.1038/nrc.2016.71 Medline

4. J. Zhang, N. N. Pavlova, C. B. Thompson, Cancer cell metabolism: The essential role of the nonessential amino acid, glutamine. EMBO J. 36, 1302-1315 (2017). doi:10.15252/embj.201696151 Medline

5. K. A. Frauwirth, J. L. Riley, M. H. Harris, R. V. Parry, J. C. Rathmell, D. R. Plas, R. L. Elstrom, C. H. June, C. B. Thompson, The CD28 signaling pathway regulates glucose metabolism. Immunity 16, 769-777 (2002). doi:10.1016/S10747613(02)00323-0 Medline

6. R. Wang, C. P. Dillon, L. Z. Shi, S. Milasta, R. Carter, D. Finkelstein, L. L. McCormick, P. Fitzgerald, H. Chi, J. Munger, D. R. Green, The transcription factor Myc controls metabolic reprogramming upon T lymphocyte activation. Immunity 35, 871-882 (2011). doi:10.1016/i.immuni.2011.09.021 Medline

7. S. Kouidhi, F. Ben Ayed, A. Benammar Elgaaied, Targeting tumor metabolism: A new challenge to improve immunotherapy. Front. Immunol. 9, 353 (2018). doi:10.3389/fimmu.2018.00353 Medline

8. S. Kouidhi, M. Z. Noman, C. Kieda, A. B. Elgaaied, S. Chouaib, Intrinsic and tumor microenvironment-induced metabolism adaptations of T cells and impact on their differentiation and function. Front. Immunol. 7, 114 (2016). doi:10.3389/fimmu.2016.00114 Medline

9. I. Shevchenko, A. V. Bazhin, Metabolic checkpoints: Novel avenues for immunotherapy of cancer. Front. Immunol. 9, 1816 (2018). doi:10.3389/fimmu.2018.01816 Medline

10. G. C. Prendergast, W. J. Malachowski, A. Mondal, P. Scherle, A. J. Muller, Indoleamine 2,3-dioxygenase and its therapeutic inhibition in cancer. Int. Rev. Cell Mol. Biol. 336, 175-203 (2018). doi:10.1016/bs.ircmb.2017.07.004 Medline

11. E. Zhao, T. Maj, I. Kryczek, W. Li, K. Wu, L. Zhao, S. Wei, J. Crespo, S. Wan, L. Vatan, W. Szeliga, I. Shao, Y. Wang, Y. Liu, S. Varambally, A. M. Chinnaiyan, T. H. Welling, 
V. Marquez, J. Kotarski, H. Wang, Z. Wang, Y. Zhang, R. Liu, G. Wang, W. Zou, Cancer mediates effector $T$ cell dysfunction by targeting microRNAs and EZH2 via glycolysis restriction. Nat. Immunol. 17, 95-103 (2016). doi:10.1038/ni.3313 Medline

12. D. B. Rivadeneira, G. M. Delgoffe, Antitumor T-cell reconditioning: Improving metabolic fitness for optimal cancer immunotherapy. Clin. Cancer Res. 24, 24732481 (2018). doi:10.1158/1078-0432.CCR-17-0894 Medline

13. C. H. Chang, J. Qiu, D. O'Sullivan, M. D. Buck, T. Noguchi, J. D. Curtis, Q. Chen, M. Gindin, M. M. Gubin, G. J. W. van der Windt, E. Tonc, R. D. Schreiber, E. J. Pearce, E. L. Pearce, Metabolic competition in the tumor microenvironment is a driver of cancer progression. Cell 162, 1229-1241 (2015). doi:10.1016/..cell.2015.08.016 Medline

14. H. Dong, T. N. Bullock, Metabolic influences that regulate dendritic cell function in tumors. Front. Immunol. 5, 24 (2014). doi:10.3389/fimmu.2014.00024 Medline

15. S. M. Davidson, T. Papagiannakopoulos, B. A. Olenchock, J. E. Heyman, M. A. Keibler, A. Luengo, M. R. Bauer, A. K. Jha, J. P. O'Brien, K. A. Pierce, D. Y. Gui, L. B. Sullivan, T. M. Wasylenko, L. Subbaraj, C. R. Chin, G. Stephanopolous, B. T. Mott, T. Jacks, C. B. Clish, M. G. Vander Heiden, Environment impacts the metabolic dependencies of Ras-driven non-small cell lung cancer. Cell Metab. 23, 517-528 (2016). doi:10.1016/i.cmet.2016.01.007 Medline

16. M. Gross, J. Chen, J. Englert, J. Janes, R. Leone, A. MacKinnon, F. Parlati, M. Rodriquez, P. Shwonek, J. Powell, Abstract 2329: Glutaminase inhibition with CB839 enhances anti-tumor activity of PD- 1 and PD-L1 antibodies by overcoming a metabolic checkpoint blocking T cell activation. Cancer Res. 76, 2329 (2016).

17. C. T. Hensley, A. T. Wasti, R. J. DeBerardinis, Glutamine and cancer: Cell biology, physiology, and clinical opportunities. J. Clin. Invest. 123, 3678-3684 (2013). doi:10.1172/JC169600 Medline

18. L. M. Pinkus, Glutamine binding sites. Methods Enzymol. 46, 414-427 (1977). doi:10.1016/S0076-6879(77)46049-X Medline

19. K. M. Lemberg, J. J. Vornov, R. Rais, B. S. Slusher, We're not "DON" yet: Optimal dosing and prodrug delivery of 6-diazo-5-oxo-L-norleucine. Mol. Cancer Ther. 17, 1824-1832 (2018). doi:10.1158/1535-7163.MCT-17-1148 Medline

20. G. Lynch, N. Kemeny, E. Casper, Phase II evaluation of DON (6-diazo-5-oxo-Lnorleucine) in patients with advanced colorectal carcinoma. Am. J. Clin. Oncol. 5 , 541-543 (1982). doi:10.1097/00000421-198210000-00014 Medline

21. R. H. Earhart, D. J. Amato, A. Y. Chang, E. C. Borden, M. Shiraki, M. E. Dowd, R. L. Comis, T. E. Davis, T. J. Smith, Phase II trial of 6-diazo-5-oxo-L-norleucine versus aclacinomycin-A in advanced sarcomas and mesotheliomas. Invest. New Drugs 8 , 113-119 (1990). doi:10.1007/BF00216936 Medline

22. R. T. Eagan, S. Frytak, W. C. Nichols, E. T. Creagan, J. N. Ingle, Phase II study on DON in patients with previously treated advanced lung cancer. Cancer Treat. Rep. 66, 1665-1666 (1982). Medline

23. G. B. Magill, W. P. L. Myers, H. C. Reilly, R. C. Putnam, J. W. Magill, M. P. Sykes, G. C. Escher, D. A. Karnofsky, J. H. Burchenal, Pharmacological and initial therapeutic observations on 6-diazo-5-oxo-1-norleucine (DON) in human neoplastic disease. Cancer 10, 1138-1150 (1957). doi:10.1002/10970142(195711/12)10:6<1138:AID-CNCR2820100608>3.0.C0;2-K Medline

24. S. Krantz, S. Rivers, R. W. Dwight, H. F. Corbus, J. Wolf, I. Green, P. W. Spear, L. T. Imperato, S. Lobe, R. M. Whittington, J. M. Rumball, A. Marquez, C. Cables, A. I. Chernoff, D. K. Misra, R. D. Sullivan, E. Miller, F. S. Dietrich, G. I. Plitman, H. P. Close, S. McCracken, A. S. Glushien, D. L. Rucknagel, C. C. Li, D. Kodlin, A clinical study of the comparative effect of nitrogen mustard and DON in patients with bronchogenic carcinoma, Hodgkin's disease, lymphosarcoma, and melanoma. J. Natl. Cancer Inst. 22, 433-439 (1959). doi:10.1093/inci/22.2.433 Medline

25. R. Rais, A. Jančařík, L. Tenora, M. Nedelcovych, J. Alt, J. Englert, C. Rojas, A. Le, A. Elgogary, J. Tan, L. Monincová, K. Pate, R. Adams, D. Ferraris, J. Powell, P. Majer, B. S. Slusher, Discovery of 6-diazo-5-oxo-I-norleucine (DON) prodrugs with enhanced CSF delivery in monkeys: A potential treatment for glioblastoma. $J$. Med. Chem. 59, 8621-8633(2016). doi:10.1021/acs.jmedchem.6b01069 Medline

26. J. Fu, E. Pacyniak, M. G. D. Leed, M. P. Sadgrove, L. Marson, M. Jay, Interspecies differences in the metabolism of a multiester prodrug by carboxylesterases. $J$. Pharm. Sci. 105, 989-995 (2016). doi:10.1002/jps.24632 Medline

27. J. Van Gelder, M. Shafiee, E. De Clercq, F. Penninckx, G. Van den Mooter, R. Kinget, $P$. Augustijns, Species-dependent and site-specific intestinal metabolism of ester prodrugs. Int. J. Pharm. 205, 93-100 (2000). doi:10.1016/S0378-
5173(00)00507-X Medline

28. JHU083 is referred to as "compound 3 " in (19) and "compound 4a" in (25).

29. X. Zhu, M. T. Nedelcovych, A. G. Thomas, Y. Hasegawa, A. Moreno-Megui, W. Coomer, V. Vohra, A. Saito, G. Perez, Y. Wu, J. Alt, E. Prchalova, L. Tenora, P. Majer, R. Rais, C. Rojas, B. S. Slusher, A. Kamiya, JHU-083 selectively blocks glutaminase activity in brain CD11b cells and prevents depression-associated behaviors induced by chronic social defeat stress. Neuropsychopharmacology 44 , 683-694 (2019). doi:10.1038/s41386-018-0177-7 Medline

30. M. T. Nedelcovych, B.-H. Kim, X. Zhu, L. E. Lovell, A. A. Manning, J. Kelschenbach, E. Hadas, W. Chao, E. Prchalová, R. P. Dash, Y. Wu, J. Alt, A. G. Thomas, R. Rais, A. Kamiya, D. J. Volsky, B. S. Slusher, Glutamine antagonist JHUO83 normalizes aberrant glutamate production and cognitive deficits in the EcoHIV murine model of HIV-associated neurocognitive disorders. J. Neuroimmune Pharmacol. 14, 391400 (2019). doi:10.1007/s11481-019-09859-w Medline

31. B. Murter, X. Pan, E. Ophir, Z. Alteber, M. Azulay, R. Sen, O. Levy, L. Dassa, I. Vaknin, T. Fridman-Kfir, R. Salomon, A. Ravet, A. Tam, D. Levin, Y. Vaknin, E. Tatirovsky, A. Machlenkin, D. Pardoll, S. Ganguly, Mouse PVRIG Has CD8+ T cell-specific coinhibitory functions and dampens antitumor immunity. Cancer Immunol. Res. 7, 244-256 (2019). doi:10.1158/2326-6066.CIR-18-0460 Medline

32. B. L. Horton, J. B. Williams, A. Cabanov, S. Spranger, T. F. Gajewski, Intratumoral CD8 ${ }^{+}$T-cell apoptosis is a major component of T-cell dysfunction and impedes antitumor immunity. Cancer Immunol. Res. 6, 14-24 (2018). doi:10.1158/23266066.CIR-17-0249 Medline

33. D. A. Chisolm, D. Savic, A. J. Moore, A. Ballesteros-Tato, B. León, D. K. Crossman, C. Murre, R. M. Myers, A. S. Weinmann, CCCTC-binding factor translates interleukin 2- and $\alpha$-ketoglutarate-sensitive metabolic changes in T cells into context-dependent gene programs. Immunity 47, 251-267.e7 (2017). doi:10.1016/j.immuni.2017.07.015 Medline

34. P. A. Tyrakis, A. Palazon, D. Macias, K. L. Lee, A. T. Phan, P. Veliça, J. You, G. S. Chia, J. Sim, A. Doedens, A. Abelanet, C. E. Evans, J. R. Griffiths, L. Poellinger, A. W. Goldrath, R. S. Johnson, S-2-hydroxyglutarate regulates CD8 ${ }^{+}$T-lymphocyte fate. Nature 540, 236-241(2016). doi:10.1038/nature20165 Medline

35. G. J. van der Windt, B. Everts, C.-H. Chang, J. D. Curtis, T. C. Freitas, E. Amiel, E. J. Pearce, E. L. Pearce, Mitochondrial respiratory capacity is a critical regulator of $\mathrm{CD}^{+} \mathrm{T}$ cell memory development. Immunity 36, 68-78 (2012). doi:10.1016/i.immuni.2011.12.007 Medline

36. M. L. Balmer, E. H. Ma, G. R. Bantug, J. Grählert, S. Pfister, T. Glatter, A. Jauch, S. Dimeloe, E. Slack, P. Dehio, M. A. Krzyzaniak, C. G. King, A.-V. Burgener, M. Fischer, L. Develioglu, R. Belle, M. Recher, W. V. Bonilla, A. J. Macpherson, S. Hapfelmeier, R. G. Jones, C. Hess, Memory $\mathrm{CD}^{+} \mathrm{T}$ cells require increased concentrations of acetate induced by stress for optimal function. Immunity $\mathbf{4 4}$. 1312-1324 (2016). doi:10.1016/i.immuni.2016.03.016 Medline

37. J. Qiu, M. Villa, D. E. Sanin, M. D. Buck, D. O'Sullivan, R. Ching, M. Matsushita, K. M. Grzes, F. Winkler, C.-H. Chang, J. D. Curtis, R. L. Kyle, N. Van Teijlingen Bakker, M. Corrado, F. Haessler, F. Alfei, J. Edwards-Hicks, L. B. Maggi Jr., D. Zehn, T. Egawa, B. Bengsch, R. I. Klein Geltink, T. Jenuwein, E. J. Pearce, E. L. Pearce, Acetate promotes $T$ cell effector function during glucose restriction. Cell Reports 27 , 2063-2074.e5 (2019). doi:10.1016/i.celrep.2019.04.022 Medline

38. S. K. Vodnala, R. Eil, R. J. Kishton, M. Sukumar, T. N. Yamamoto, N.-H. Ha, P.-H. Lee, M. Shin, S. J. Patel, Z. Yu, D. C. Palmer, M. J. Kruhlak, X. Liu, J. W. Locasale, J. Huang, R. Roychoudhuri, T. Finkel, C. A. Klebanoff, N. P. Restifo, T cell stemness and dysfunction in tumors are triggered by a common mechanism. Science 363 eaau0135 (2019). doi:10.1126/science. aau0135 Medline

39. S. Jitrapakdee, A. Vidal-Puig, J. C. Wallace, Anaplerotic roles of pyruvate carboxylase in mammalian tissues. Cell. Mol. Life Sci. 63, 843-854 (2006). doi:10.1007/s00018-005-5410-y Medline

40. T. Cheng, J. Sudderth, C. Yang, A. R. Mullen, E. S. Jin, J. M. Matés, R. J. DeBerardinis, Pyruvate carboxylase is required for glutamine-independent growth of tumor cells. Proc. Natl. Acad. Sci. U.S.A. 108, 8674-8679 (2011). doi:10.1073/pnas.1016627108 Medline

41. K. Sellers, M. P. Fox, M. Bousamra II, S. P. Slone, R. M. Higashi, D. M. Miller, Y. Wang, J. Yan, M. O. Yuneva, R. Deshpande, A. N. Lane, T. W.-M. Fan, Pyruvate carboxylase is critical for non-small-cell lung cancer proliferation. J. Clin. Invest. 125, 687-698 (2015). doi:10.1172/JC172873 Medline

42. M. St. Maurice, L. Reinhardt, K. H. Surinya, P. V. Attwood, J. C. Wallace, W. W. 
Cleland, I. Rayment, Domain architecture of pyruvate carboxylase, a biotindependent multifunctional enzyme. Science 317, 1076-1079 (2007). doi:10.1126/science.1144504 Medline

43. M. F. Utter, D. B. Keech, Pyruvate carboxylase. I. Nature of the reaction. J. Biol. Chem. 238, 2603-2608 (1963). Medline

44. F. R. Dejure, N. Royla, S. Herold, J. Kalb, S. Walz, C. P. Ade, G. Mastrobuoni, J. T. Vanselow, A. Schlosser, E. Wolf, S. Kempa, M. Eilers, The MYC mRNA 3'-UTR couples RNA polymerase II function to glutamine and ribonucleotide levels. EMBO J. 36, 1854-1868 (2017). doi:10.15252/embj.201796662 Medline

45. B. Faubert, G. Boily, S. Izreig, T. Griss, B. Samborska, Z. Dong, F. Dupuy, C. Chambers, B. J. Fuerth, B. Viollet, O. A. Mamer, D. Avizonis, R. J. DeBerardinis, P. M. Siegel, R. G. Jones, AMPK is a negative regulator of the Warburg effect and suppresses tumor growth in vivo. Cell Metab. 17, 113-124 (2013). doi:10.1016/i.cmet.2012.12.001 Medline

46. H. M. Haikala, J. M. Anttila, J. Klefström, MYC and AMPK-Save energy or die! Front. Cell Dev. Biol. 5, 38 (2017). doi:10.3389/fcell.2017.00038 Medline

47. J. W. Kim, C. V. Dang, Cancer's molecular sweet tooth and the Warburg effect. Cancer Res. 66, 8927-8930 (2006). doi:10.1158/0008-5472.CAN-06-1501 Medline

48. A. B. Kohan, I. Talukdar, C. M. Walsh, L. M. Salati, A role for AMPK in the inhibition of glucose-6-phosphate dehydrogenase by polyunsaturated fatty acids. Biochem. Biophys. Res. Commun. 388, 117-121 (2009). doi:10.1016/j.bbrc.2009.07.130 Medline

49. Z. E. Stine, Z. E. Walton, B. J. Altman, A. L. Hsieh, C. V. Dang, MYC, metabolism, and cancer. Cancer Discov. 5, 1024-1039 (2015). doi:10.1158/2159-8290.CD-150507 Medline

50. O. Warburg, K. Gawehn, A. W. Geissler, Stoffwechsel der weissen Blutzellen. Z. Naturforsch. B 13, 515-516 (1958). doi:10.1515/znb-1958-0806 Medline

51. A. Bakker, Einige Übereinstimmungen im Stoffwechsel der Carcinomzellen und Exsudatleukocyten. Klin. Wochenschr. 6, 252-254 (1927). doi:10.1007/BF01710710

52. V. A. Gerriets, J. C. Rathmell, Metabolic pathways in T cell fate and function. Trends Immunol. 33, 168-173 (2012). doi:10.1016/j.it.2012.01.010 Medline

53. N. J. Maclver, R. D. Michalek, J. C. Rathmell, Metabolic regulation of $T$ lymphocytes. Annu. Rev. Immunol. 31, 259-283 (2013). doi:10.1146/annurevimmunol-032712-095956 Medline

54. E. L. Pearce, M. C. Poffenberger, C. H. Chang, R. G. Jones, Fueling immunity: Insights into metabolism and lymphocyte function. Science 342, 1242454(2013). doi:10.1126/science.1242454 Medline

55. P. J. Siska, K. E. Beckermann, F. M. Mason, G. Andrejeva, A. R. Greenplate, A. B. Sendor, Y. J. Chiang, A. L. Corona, L. F. Gemta, B. G. Vincent, R. C. Wang, B. Kim, J. Hong, C. L. Chen, T. N. Bullock, J. M. Irish, W. K. Rathmell, J. C. Rathmell, Mitochondrial dysregulation and glycolytic insufficiency functionally impair CD8 T cells infiltrating human renal cell carcinoma. JCl Insight 2, e93411 (2017). doi:10.1172/jci.insight.93411 Medline

56. M. V. Liberti, J. W. Locasale, The Warburg effect: How does it benefit cancer cells? Trends Biochem. Sci. 41, 211-218 (2016). doi:10.1016/j.tibs.2015.12.001 Medline

\section{ACKNOWLEDGMENTS}

We thank W. Xu, E. Thompson, S. Collins, R. Helms, M. Claiborne and other members of the Powell lab for critical discussion of the manuscript. Funding: This work was supported by the National Institutes of Health (R01CA226765 to JDP, R01CA229451 to JDP and BSS) and The Bloomberg Kimmel Institute for Cancer Immunotherapy. Author contributions: RDL and JME, designed and conducted tumor growth and survival experiments. RDL, LZ, JME, IMS, MHO, HIS, MLA, IAB, CHP, JW, AT, RLB, JA all were involved in performing experiments. EP, RR performed drug synthesis. BSS supervised drug synthesis and development. RDL and JDP designed and supervised experiments. RDL and JDP wrote the manuscript. Competing interests: JDP, BS, RR and PM are scientific founders, paid consultants (JDP, BS) and have equity in Dracen Pharmaceuticals. Technology arising in part from the studies described herein were patented by Johns Hopkins University and subsequently licensed to Dracen Pharmaceuticals (JHU083 is currently labeled as DRP-083). RDL, JME, BS, RR, $J A$, and JDP are inventors for pending patent application no. PCT/US16/44829, submitted by Johns Hopkins University that covers the use of glutamine analogs, such as JHU083 (DRP-083) for cancer immunotherapy. Data and materials availability: The chemical structure of JHU083 ((Ethyl 2-(2-Amino-4methylpentanamido)-DON) has previously been published as "prodrug 4a" (25) and "compound 3" (19). RNA seq data has been deposited in the National Institutes of Health (NIH) Gene Expression Omnibus (GEO) repository, accession \#GSE120345.

\section{SUPPLEMENTARY MATERIALS}

science.sciencemag.org/cgi/content/full/science.aav2588/DC1

Materials and Methods

Figs. S1 to S7

References

Data S1

23 September 2018; resubmitted 21 July 2019

Accepted 25 October 2019

Published online 7 November 2019

10.1126/science.aav2588 
A.
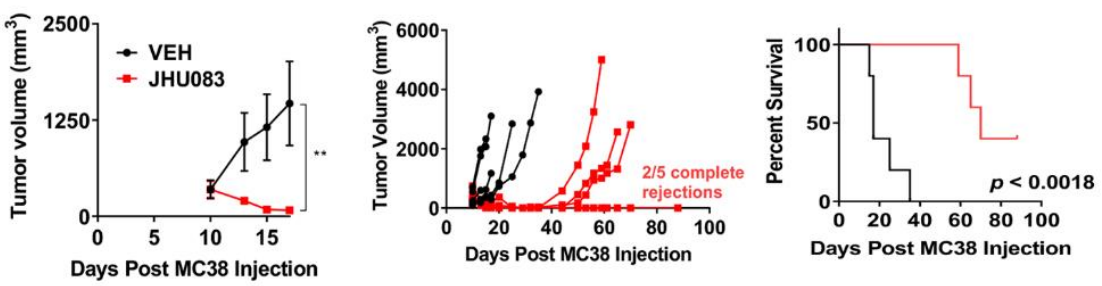

B.
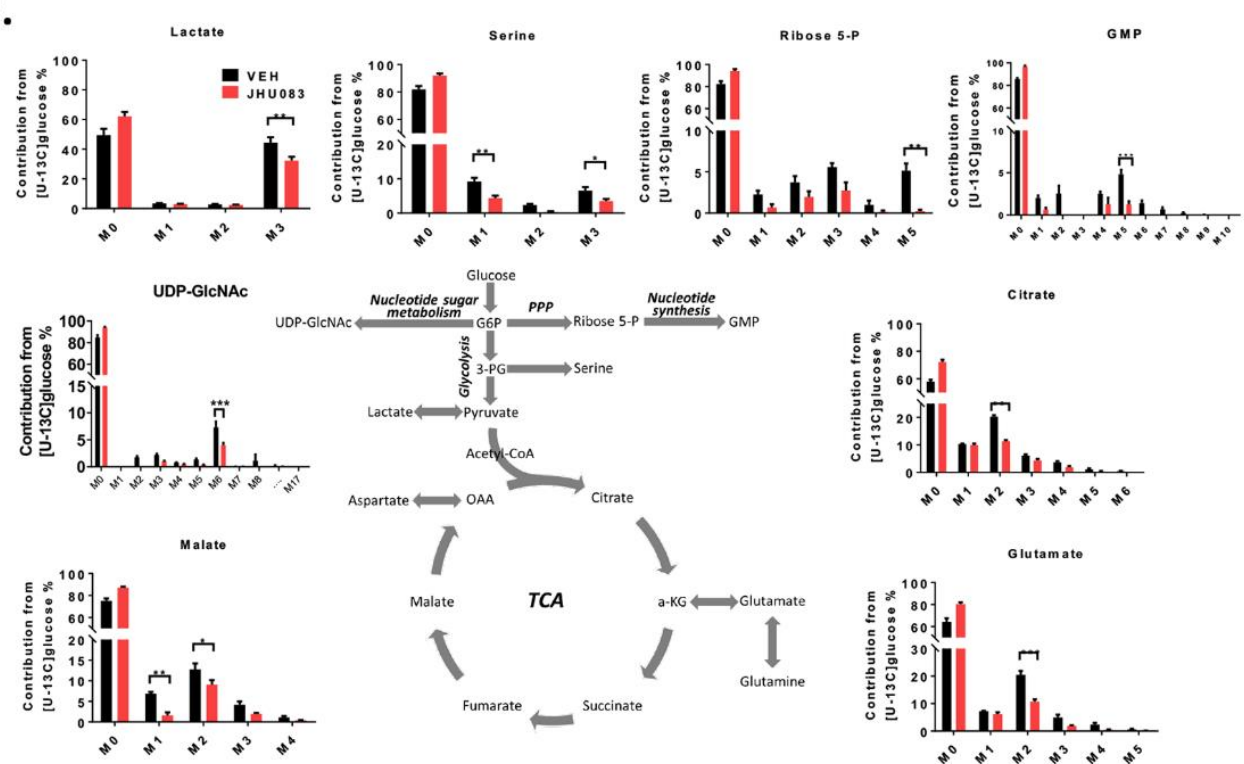

C.

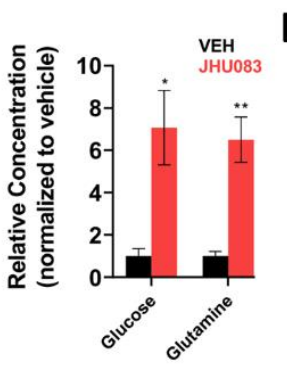

D.

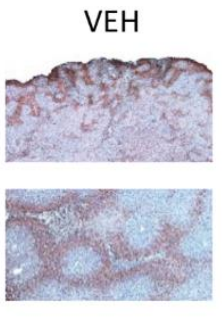

F.

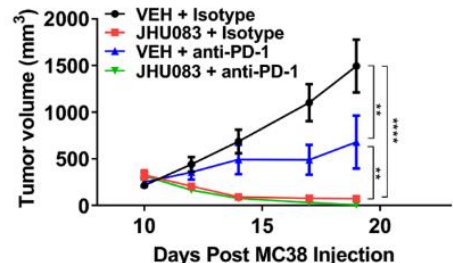

H.

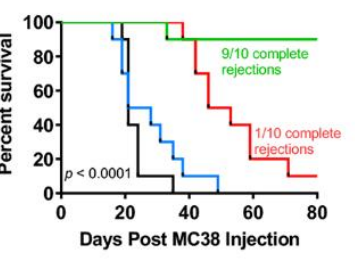

JHU083
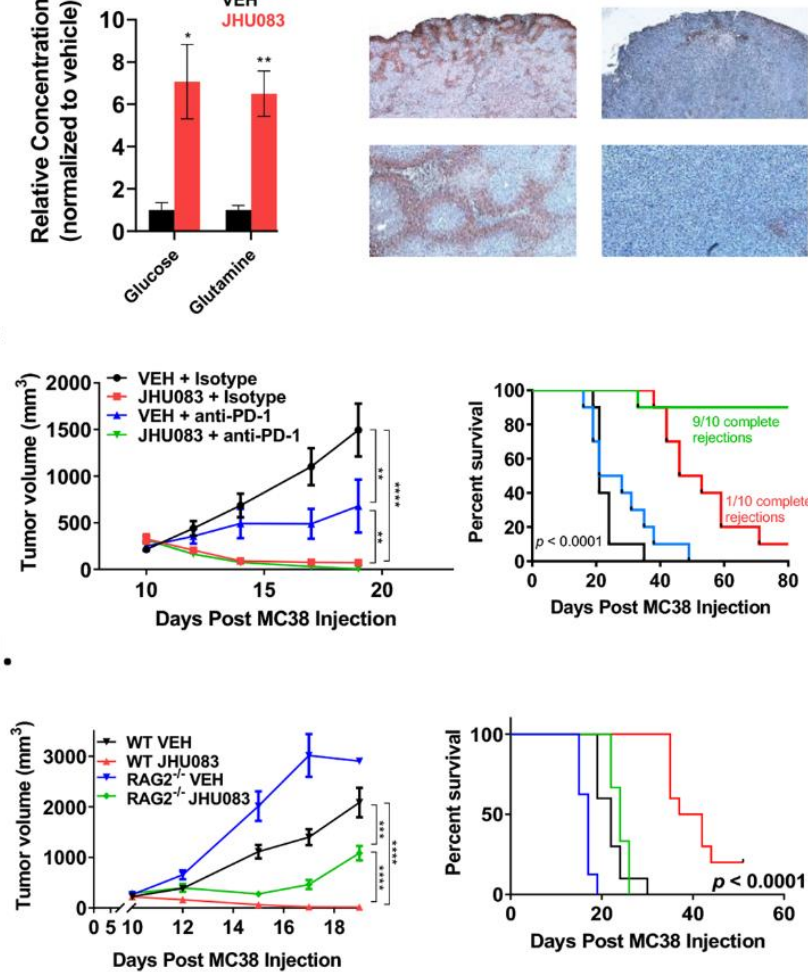

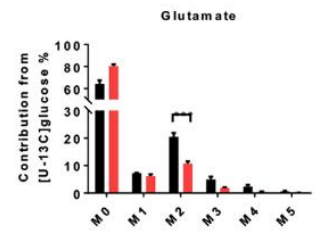

E.

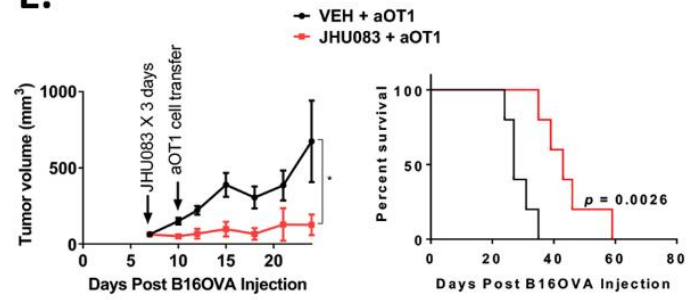

G.

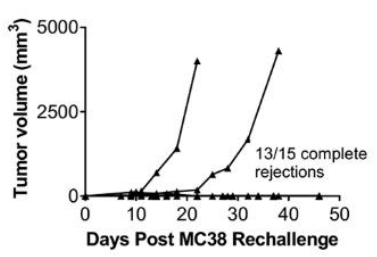

I.
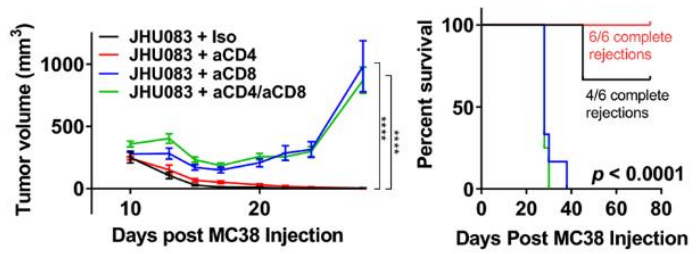
Fig. 1. Glutamine blockade suppresses cancer cell metabolic programs and enhances antitumor immune response. (A) Average tumor growth curve (left), spider plots (center) and survival curve (right) from vehicle and JHU083 treated MC38-bearing mice. (B) In vivo ${ }^{13} \mathrm{C}$-glucose tracing experiment in MC38 tumor-bearing mice; $M$, unlabeled mass of isotope; $M+n$, native metabolite mass + number of isotopically labeled carbons. (C) Relative mass spectrometric quantification of glucose and glutamine in MC38 tumors from vehicle and JHU083 treated mice (per mg tumor tissue, normalized to vehicle group). (D) Pimonidazole IHC staining for hypoxia in tumor sections from vehicle and JHU083 treated mice. (E) B160VA-bearing C57BL/6 mice treated with JHU083 or vehicle on days 7-9 after tumor inoculation received $1.5 \times 10^{6}$ activated OTI T cells on day 10 . Tumor growth curve (left) and survival curve (right). (F) MC38 bearing C57BL/6 mice treated with vehicle, JHU083, anti-PD-1, or combination JHU083 and anti-PD-1 beginning on day 10 after tumor inoculation. Tumor growth curve and survival curve are shown. (G) Mice initially cured with 14 days of JHU083 treatment were rechallenged $\geq 30$ days after last dose of JHU083; spider plots of tumor volume shown. $(\mathrm{H}) \mathrm{MC} 38$-bearing $\mathrm{C} 57 \mathrm{BL} / 6$ wild type and RAG2 ${ }^{-/-}$mice treated with 14 days of vehicle or JHU083; average tumor volume (until first sacrifice in WT VEH group) and survival curve shown. (I) MC38 bearing C57BL/6 mice treated with JHU083 after depletion of CD8 cells, CD4 cells, or both compared with isotype control. Tumor growth curve and survival curve are shown. Error bars represent SEM. Data are representative of one (B), three $[(E)$ and $(F)]$, or five (D) independent experiments with $n=3-10$ per group. Tumor growth curves were assessed by two-way ANOVA. Logrank (Mantel-Cox) tests were performed for survival data. Metabolite data assessed with two-tailed student's $t$ test for multiple comparisons. ${ }^{*} p<0.05,{ }^{*} p<0.01,{ }^{* *} p<0.001$ using two-tailed Student's $t$ test. 


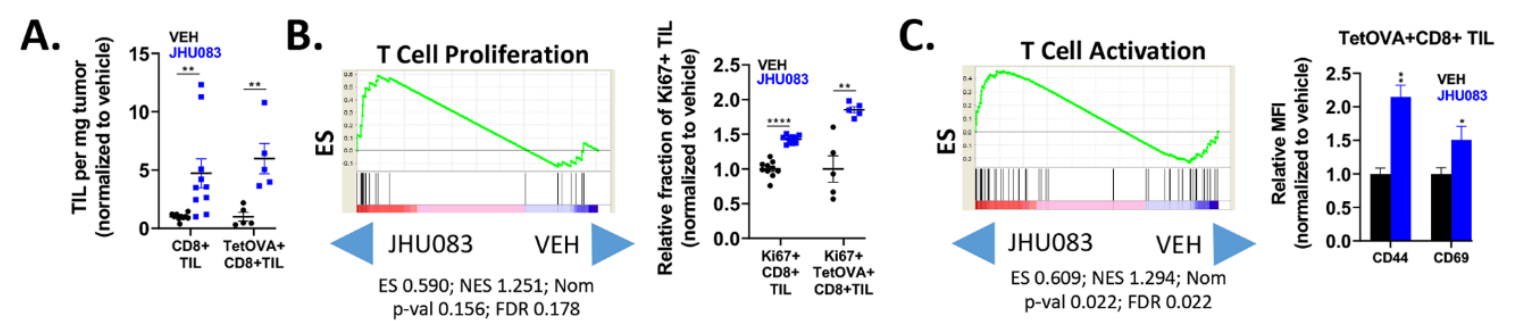

D.
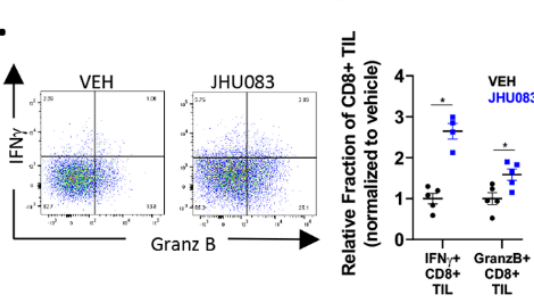

E.

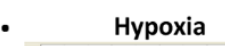

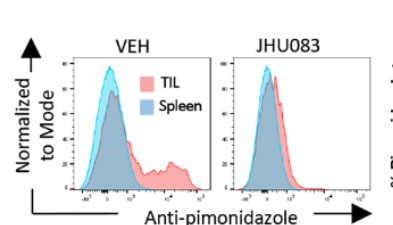
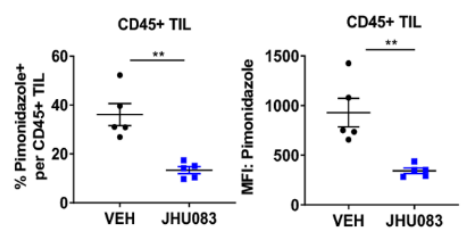

F.

Memory vs Effector

CD8 T Cell

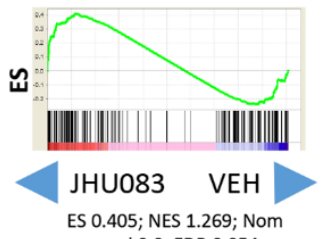

ES 0.405; NES 1.269; Nom
p-val 0.0; FDR 0.054

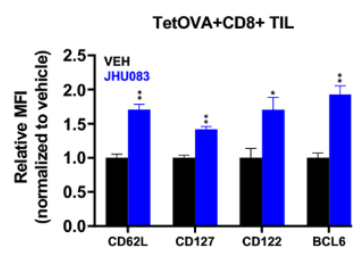

H.

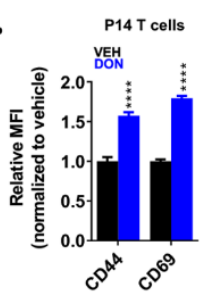

I.

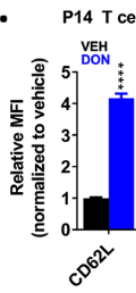

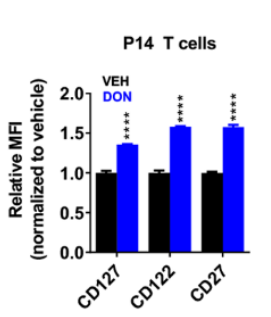

J.

G.
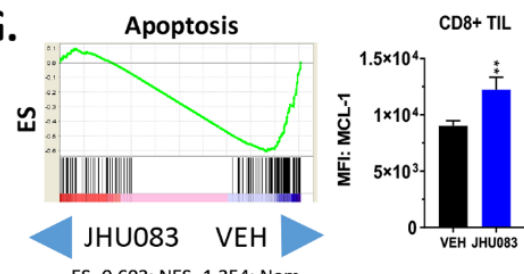

ES -0.603; NES -1.354; Nom

p-val 0.0; FDR 0.010
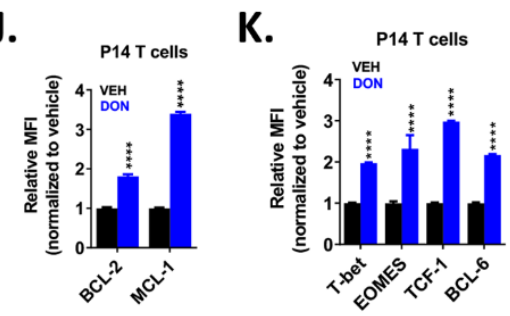

L.
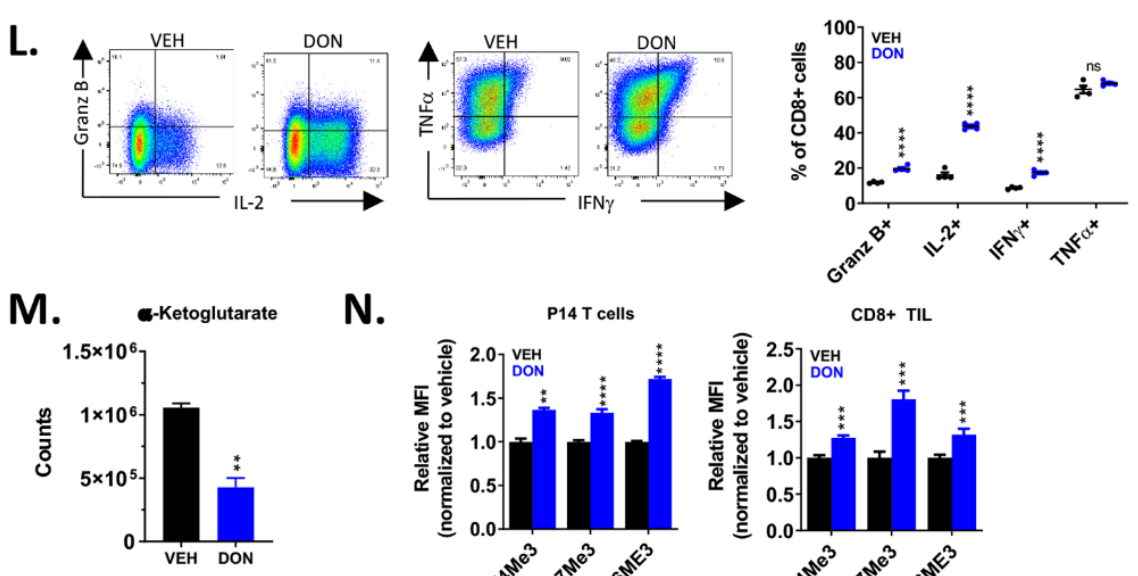

N.

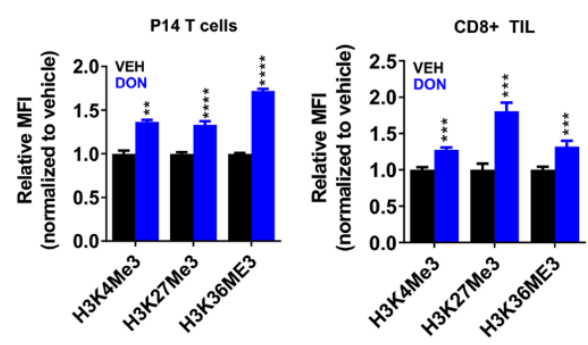


Fig. 2. Glutamine blockade conditions CD8+ TIL toward a highly proliferative, activated and long-lived phenotype. C57BL/6 mice with MC38 or MC380VA tumors were treated on days 14-18 with vehicle or JHU083 $(0.3 \mathrm{mg} / \mathrm{kg} /$ day $)$ and sacrificed on day 18 for analysis. (A) Percentage live CD8+ or TetOVA+CD8+ TIL per tumor weight for MC38 and MC380VA models, respectively. (B) GSEA tracing for positive regulation of $\alpha \beta$ T cell proliferation (left); percentage Ki67+ cells per CD8+ in MC38 model or TetOVA+CD8+ in MC380VA model (right). (C) GSEA tracing for $\alpha \beta$ T cell activation (left); mean fluorescence intensity (MFI) of CD44 and CD69 of TetOVA+CD8+ TIL in MC380VA model (right). (D) Relative fraction (normalized to vehicle) of IFN $\gamma+$ and Granzyme B + per CD8+ TIL from the MC380VA model after ex vivo stimulation with SIINFEKL peptide for 4 hours. (E) GSEA tracing for hypoxic exposure (left); FACS plots (center) data summary (right) showing percentage of pimonidazole positive and pimonidazole MFI of CD45+ TIL in the MC38 model. (F) GSEA tracings for memory vs KLRG1high effector CD8 T cells (left); relative MFI of CD62L, CD127, CD122 and BCL-6 of TetOVA+CD8+ TIL (right). (G) GSEA tracing for apoptosis (left); MFI of MCL-1 on CD8+ in MC38 model (center); live cell percentage of CD8+ TIL in MC38 model and TetOVA+CD8+ in MC380VA model (right). (H to K) Naïve P14 T cells activated in the presence of vehicle or DON $(1 \mu \mathrm{M})$ for 2 days, rested in IL-2 for 2 additional days with vehicle or DON and analyzed by FACS for activation markers $(\mathrm{H})$, memory markers $(\mathrm{I})$, survival markers $(\mathrm{J})$ and transcription factors (K). (L) P14 T cells activated for 2 days as described, rested for 4 days in the presence of vehicle or DON before re-stimulation (with no drug present) for 4 hours: flow plots and data summary for intracellular cytokines are shown. (M) Relative $\alpha$-ketoglutarate levels at 36 hours post P14 activation. (N) H3 histone trimethylation levels in CD8+ TIL (left) and P14 T cells 3 days post activation (right). Error bars represent SEM. For MC380VA experiments, FACS data are representative of 2-3 independent experiments with $n=5$ mice per group. For MC38 experiments FACS summary plots are combination data from 2 independent experiments with $n=5$ mice per group. For RNA-seq data are from treated and untreated groups of 5 mice in each group. In vitro experiments are representative of 3-5 independent experiments with 3-6 per group. ${ }^{*} p<0.05$, ${ }^{* *} p<0.01,{ }^{* *} p<0.001$ using two-tailed Student's $t$ test. 
A.

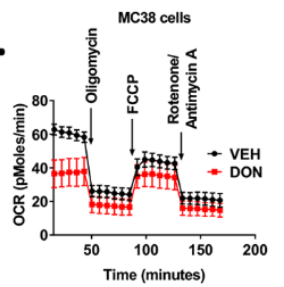

B.

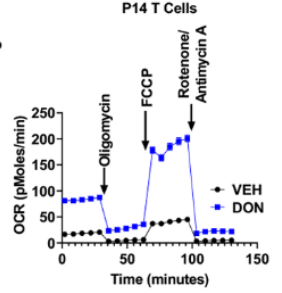

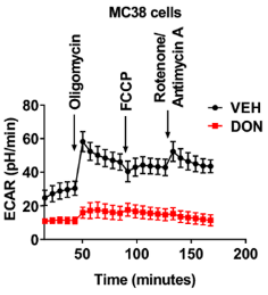

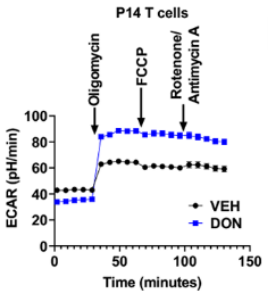

G.

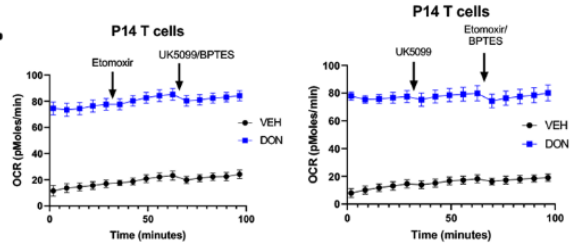

I.

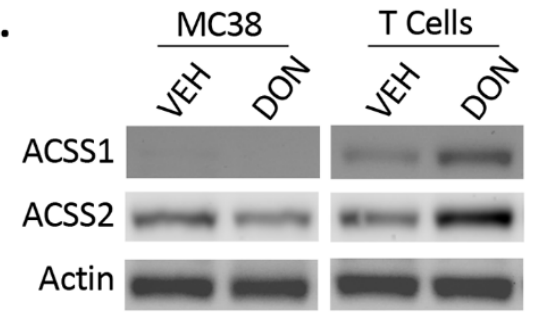

J.
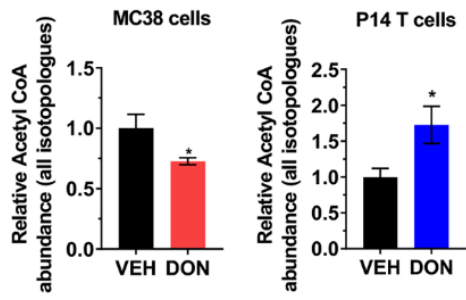

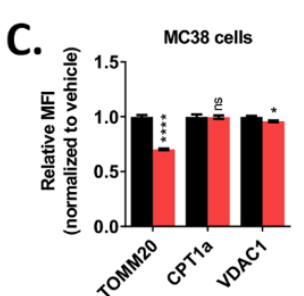

D.

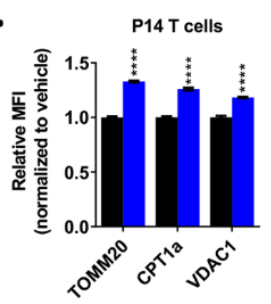

F.
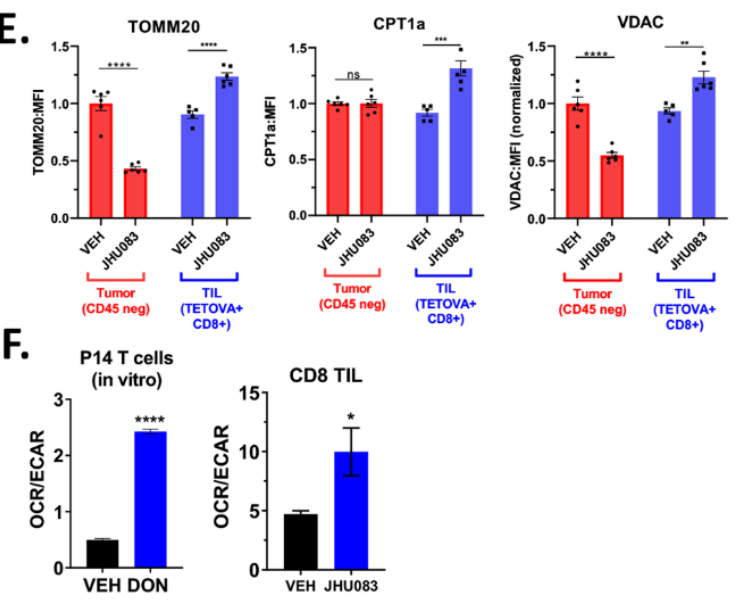

H.
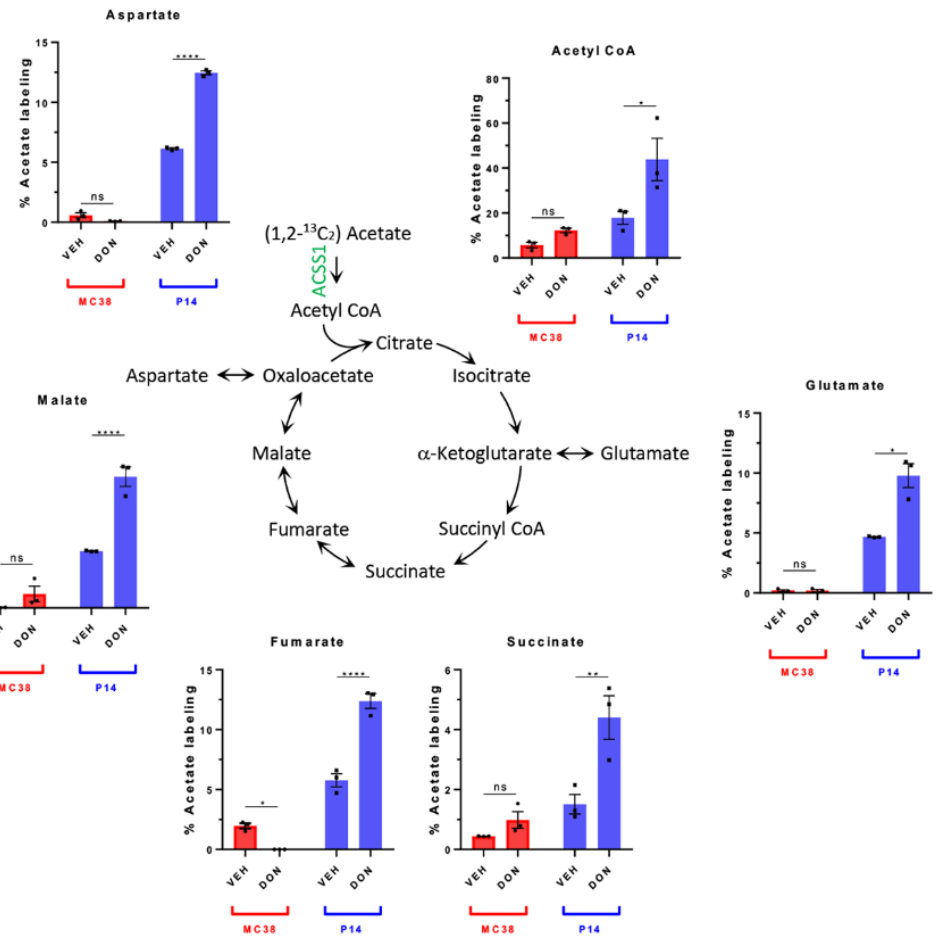

Fig. 3. Activated CD8+ T cells and MC38 tumor cells enact distinct metabolic programs in response to glutamine antagonist treatment. (A to D) Differential metabolic characteristics of vehicle treated vs. DON treated MC38 cells and vehicle treated vs. DON treated activated T CD8+ T cells in vitro. Extracellular acidification rate (ECAR) and oxygen consumption rate (OCR) from metabolic flux analyses [(A) and (B)]. Relative expression of mitochondrial proteins [(C) and (D)]. (E) Differential expression of mitochondrial proteins in CD8+ TIL and CD45-negative tumor cells from explanted MC38 tumors. (F) OCR/ECAR ratio in vehicle- and DON-treated activated CD8 in vitro (left), and in CD8+ TIL harvested from MC38 murine tumors after vehicle or JHU083 treatment (right). (G) OCR response to etomoxir, UK5099, and BPTES in vehicle- vs. DON-treated activated P14 CD8 in vitro. Vehicle treated vs. DON treated MC38 cells and vehicle and DON treated activated T CD8+ T cells in vitro. (H) LCMS analysis of TCA intermediates after stable isotope tracing with [1,213C] acetate. (I) Western blot analysis of ACSS1 and ACSS2 expression. (J) LCMS analysis of relative acetyl-CoA abundance. Error bars represent SEM. Data are representative of three $[(A),(C),(D),(E)$, $(G)$, and $(I)]$ or six $[(B)$ and $(F)]$ independent experiments with $n=3-8$ per group. ${ }^{* *} p<0.001$, $*^{* * *} p<0.0001$ using two-tailed Student's $t$ test. 
A.

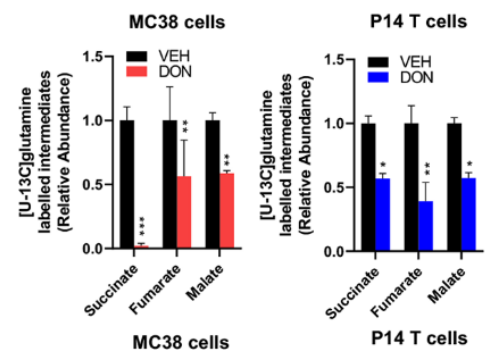

B.

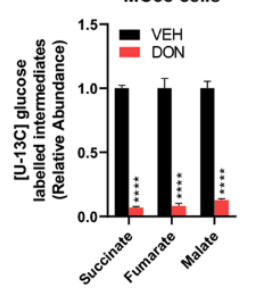

D.

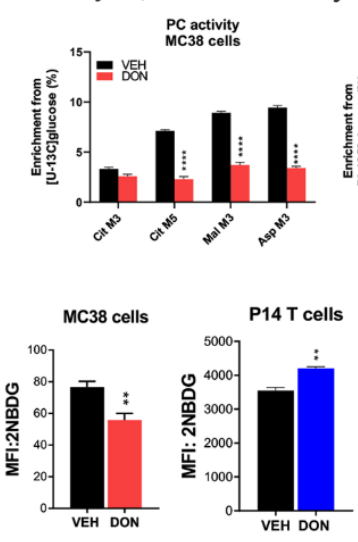

C.
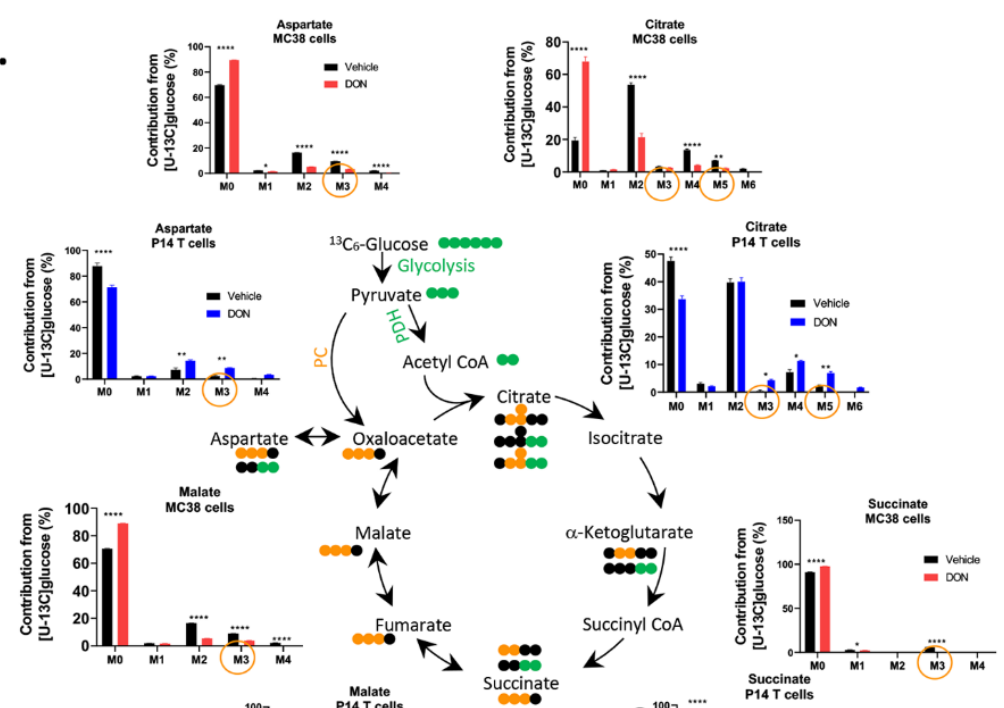

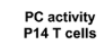

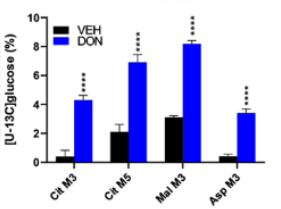

F. Glycolysis intermediates
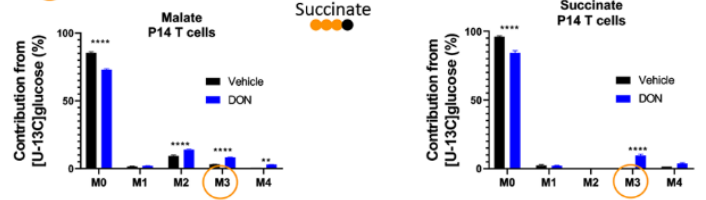

G.
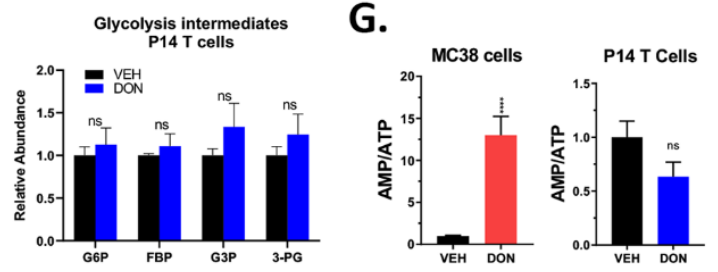
H. $\frac{\mathrm{MC} 38}{\mathrm{VEH} \text { DON }} \frac{\mathrm{P} 14}{\mathrm{VEH} \text { DON }}$
c-MYC $=-1$
p-AMPK - -
AMPK - - -
ACTIN - -
$\longrightarrow$

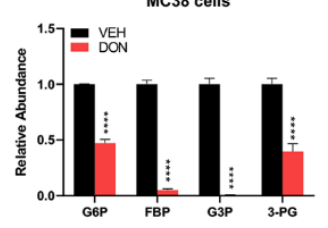

I.

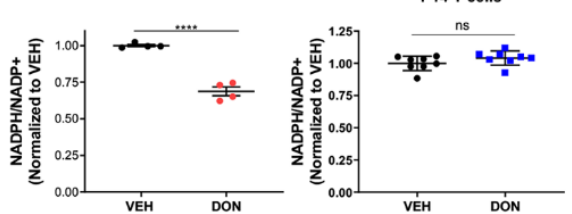

J.

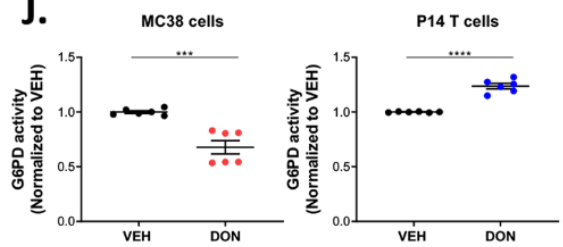

K.

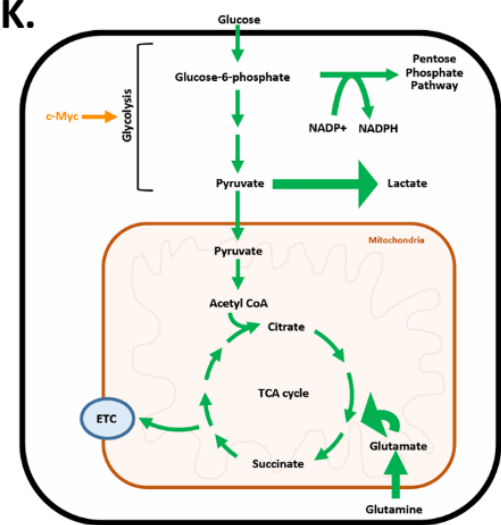

Proliferative Cell Metabolism

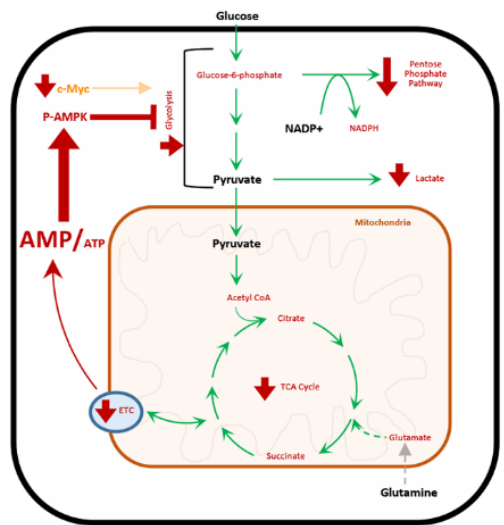

MC38 + Glutamine Antagonist

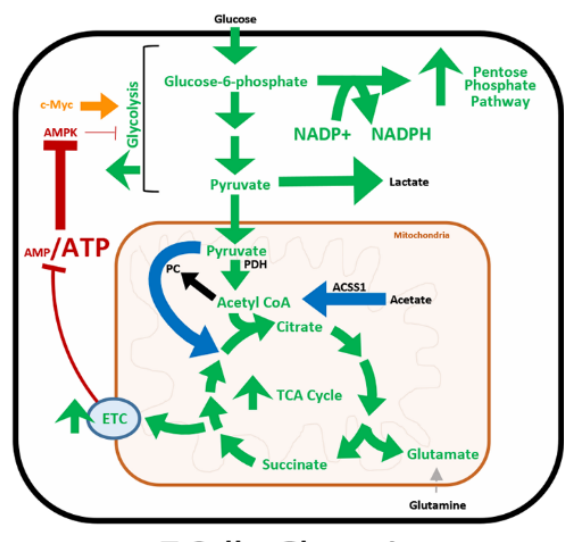

T Cell + Glutamine Antagonist 
Fig. 4. In response to glutamine blockade, activated CD8+ T cells, but not MC38 tumor cells, replenish TCA intermediates by upregulating glucose anaplerosis. (A to $\mathrm{J}$ ) Metabolic characteristics of vehicle vs. DON treated MC38 cells (red) and vehicle vs. DON treated activated T CD8+ T cells (blue) in vitro. Relative abundance of labeled intermediates of the TCA cycle during [U13C] glutamine (A) and [U-13C] glucose (B) tracing experiments. LCMS analysis of TCA intermediates [U-13C] glucose tracing (C). Relative labeling of TCA isotopologues characteristic of pyruvate carboxylase (PC) activity (D). 2-NBDG uptake by flow cytometry analysis (E). Relative abundance of glycolytic intermediates in MC38 (left) and P14 T cells (right) (F); G6P, glucose-6phosphate; FDP, fructose-1,6-bis-phosphate; G3P, D-glyceraldehyde 3-phosphate; 3-PG, D-glycerate 3-phosphate. Relative AMP/ATP ratio (G). Western blot of c-MYC, phospho-AMPK, and total AMPK expression (H). Relative NADP+/NADPH ratio (I). Relative Glucose-6-phosphate dehydrogenase (G6PD) activity $(\mathrm{J})$. Error bars represent SEM. Data are representative of 3-4 independent experiments $[(E),(H),(I)$, and $(J)]$ with $n=3-4[(E),(I)$, and $(J)] .(G)$ is abundance data compiled from three independent tracing studies. ${ }^{* *} \mathrm{p}<0.001$, ${ }^{* * *} \mathrm{p}<0.0001$ using two-tailed Student's t test. (K) Model depicting relative activity of fundamental metabolic pathways in highly proliferative cells (left), glutamine inhibited MC38 cancer cells (center) and glutamine inhibited effector CD8+ T cells (right). Highly proliferative cells in nutrient rich microenvironments engage high levels of aerobic glycolysis (Warburg physiology), glutaminolysis, and pentose phosphate pathway activity to maintain energy, redox, and metabolite homeostasis. Disruption of glutamine metabolism in MC38 cells leads to increased AMP/ATP ratio and decreased c-MYC such that proximal glycolytic metabolism is suppressed, and cells can no longer rely on Warburg physiology, the pentose phosphate pathway (PPP), or TCA cycle activity. In contrast, activated T cells adapt to glutamine blockade and maintain redox and energy homeostasis by upregulating OXPHOS through acetate catabolism, generating high levels of acetyl-CoA as a 2-carbon source for the TCA cycle and upregulating pyruvate carboxylase for glucose anaplerosis. 